\title{
Reverse Engineering Human Mobility in Large-scale Natural Disasters
}

\author{
Milan Schmittner, Max Maass, Tom Schons, and Matthias Hollick \\ Secure Mobile Networking Lab \\ Technische Universität Darmstadt \\ Darmstadt, Germany \\ firstname.lastname@seemoo.tu-darmstadt.de
}

\begin{abstract}
Delay/Disruption-Tolerant Networks (DTNs) have been around for more than a decade and have especially been proposed to be used in scenarios where communication infrastructure is unavailable. In such scenarios, DTNs can offer a best-effort communication service by exploiting user mobility. Natural disasters are an important application scenario for DTNs when the cellular network is destroyed by natural forces. To assess the performance of such networks before deployment, we require appropriate knowledge of human mobility.

In this paper, we address this problem by designing, implementing, and evaluating a novel mobility model for large-scale natural disasters. Due to the lack of GPS traces, we reverse-engineer human mobility of past natural disasters (focusing on 2010 Haiti earthquake and 2013 Typhoon Haiyan) by leveraging knowledge of 126 experts from 71 Disaster Response Organizations (DROs). By means of simulation-based experiments, we compare and contrast our mobility model to other well-known models, and evaluate their impact on DTN performance. Finally, we make our source code available to the public.
\end{abstract}

\section{KEYWORDS}

Mobility model, disaster response, DTN

\section{INTRODUCTION}

Around the globe, we observe a continuous increase in natural disaster occurrences [11]. When a disaster strikes, the communication infrastructure is often destroyed or unavailable in the immediate aftermath which hinders effective disaster relief work [5, 7].

In the humanitarian sector, messaging applications have proved extremely helpful for both organizations as well as individuals as they facilitate coordination and broadcasting information to the public [6]. Smartphone-based DTNs present themselves as an attractive technology for message-based communication in disaster scenarios where the communication infrastructure is disrupted or destroyed as these devices are readily available [17]. DTNs are facilitated by user mobility as user devices act as data mules to carry messages from $a$ to $b$. Clearly, the underlying human mobility greatly affects DTNs performance as disconnected network partitions are unable to communicate.

MSWiM '17, November 21-25, 2017, Miami, FL, USA

(C) 2017 Association for Computing Machinery.

This is the author's version of the work. It is posted here for your personal use. Not for redistribution. The definitive Version of Record was published in Proceedings of MSWiM '17, November 21-25, 2017, https://doi.org/10.1145/3127540.3127542.
Current works on DTNs usually conduct simulation-based performance evaluations but are lacking realistic mobility models for large-scale natural disasters. This lack can be attributed to the unavailability of public mobility traces due to security and privacy concerns of cellular network operators as well as DROs. In this paper, we approach the problem by creating a disaster scenario mobility model based on expert knowledge. In particular, we make the following contributions which are relevant for both the opportunistic and ad hoc network as well as the disaster response communities:

- We model the mobility of disaster response teams as well as the local population in real large-scale natural disasters. Our model is based on expert knowledge, such as operational reports and conducted interviews, gathered from 126 individuals from 71 DROs.

- We characterize our mobility model and compare it with two other widely used models via simulation. In addition, we demonstrate the impact of mobility on DTN performance.

- We provide an open source implementation [23] of our Natural Disaster mobility model as well as two exemplary scenarios: the 2010 Haiti earthquake [5] and the 2013 Typhoon Haiyan [7].

The rest of the paper is structured as follows: we first revisit the state-of-the-art of mobility models and DTN simulation in Section 2. We explain our approach in Section 3. Then, we present our mobility model and scenario design in Section 4 and their implementation in Section 5. We analyze and evaluate the impact of our mobility model in Section 6 and, finally, conclude in Section 7.

\section{RELATED WORK}

Generic mobility models such as Random Waypoint (RWP) have been used to study the performance of mobile wireless networks such as DTNs. However, these models do not capture the nonrandomness of human mobility and, therefore, produce questionable results when trying to understand network performance in realistic scenarios. To alleviate this problem, multiple works (see Table 1) have sought to create domain-specific models: [2] proposes Disaster Area which models disaster scenarios based on traces collected at a fire fighter manoeuvre. This model has been used multiple times to evaluate performance of mobile networks, e. g., [12, 20]. [10] proposes a micro-movement pattern generation framework for search-and-rescue missions. [9] states the need for expert knowledge to create realistic scenarios. [25] is closest related to our work as they attempt to model a large-scale post-disaster scenario. However, they claim that "no documents/reports happen to describe the 
Table 1: Related publications on disaster and emergency response mobility models. (*) indicates a work that proposes a new model. Our work is printed in bold.

\begin{tabular}{crrrl}
\hline Work & Nodes & Area $\left(\mathrm{m}^{2}\right)$ & Duration & Mobility Model \\
\hline$[16]$ & 100 & 1000 & $25 \mathrm{~min}$ & Event-Role $*$ \\
{$[2]$} & 150 & $350 \times 200$ & $27.7 \mathrm{~h}$ & Disaster Area $*$ \\
{$[12]$} & $\leq 80$ & $700 \times 600$ & $10 \mathrm{~min}$ & Disaster Area [2] \\
{$[1]$} & $\leq 200$ & $\leq 550 \times 500$ & $30 \mathrm{~min}$ & Disaster Area [2] \\
{$[20]$} & 200 & $550 \times 500$ & $5 \mathrm{~min}$ & Disaster Area [2] \\
{$[9]$} & 150 & $350 \times 200$ & $1.1 \mathrm{~h}$ & Disaster Area [2] \\
{$[10]$} & 100 & $6000 \times 5000$ & $35 \mathrm{~h}$ & Search \& Rescue $*$ \\
{$[25]$} & 234 & $\mathrm{~N} / \mathrm{A}$ & 4 days & Natural Disaster $*$ \\
- & $\mathbf{5 0 0}$ & $\mathbf{5 0 0 0} \times \mathbf{7 0 0 0}$ & $\mathbf{7}$ days & Natural Disaster $*$ \\
\hline
\end{tabular}

disaster operation in as detail as required to reproduce the scene by a simulator." In our work, we tackle precisely this problem and reverse-engineer human mobility at a granularity-level suitable for a simulator by using information from public and non-public documents and by conducting interviews with various International Disaster Response (IDR) experts.

\section{METHODOLOGY}

GPS mobility traces are the "holy grail" for creating accurate mobility models. Unfortunately, such ground truth data during and post disaster especially from Disaster Response Teams (DRTs) are not (publicly) available. This is due to the plain lack of records, strict data privacy laws, mobile network operators protecting their valuable business assets, as well as security concerns of DROs who do not wish to disclose sensitive location information of their staff, especially when operating in armed-conflict areas.

As a consequence, we follow an alternative approach: we base our mobility model on "soft" data, in particular, expert knowledge of various DROs. In order for this method to yield acceptable results, we need to ensure that (1) the model input is sufficiently finegrained for extracting mobility patterns, (2) the model is verified by a group of experts.

Unfortunately, documentation of the events during a disaster is often incomplete: media coverage of high-profile disaster is vast but articles typically lack technical details. Also, first responders and

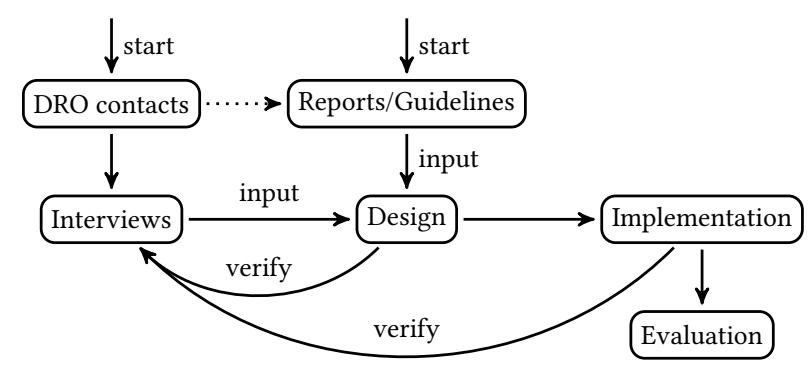

Figure 1: Our process for information gathering, model design and implementation, verification, and evaluation. disaster relief workers are supposed to create daily reports but can often invest only minimal time as they prioritize actual life-saving work. This often leads incomplete or imprecise documentation unusable for our purposes. To understand IDR and eventually derive our mobility model, we gathered information from

(1) DRO policies, guidelines, strategies and fieldbooks partly gathered from online disaster response platforms, and

(2) individual (on-site) interviews with disaster relief experts.

Our approach is visualized in Fig. 1. During the process, we contacted 298 and received feedback from 126 IDR experts (42\% response rate). From the responders, we received additional information about their humanitarian activities, past mission reports, and pointers to other experts and contacts better suited for replying to our inquiry. In addition, we received access to exclusive online platforms and forums such as the Virtual On-Site Operations Coordination Centre (VOSOCC) of the UN Office for the Coordination of Humanitarian Affairs (OCHA), reliefweb.int, and humanitarianresponse.info. They contain field handbooks and guidelines for in-field operations which are not publicly accessible. Furthermore, we were able to conduct 15 on-site interviews with IDR experts and former disaster relief workers. Those experts were also involved in the verification loop from design to implementation.

\section{DESIGN}

In this section, we review past candidate events which qualify for our methodology. We then provide a detailed time-line of events for one recent high-profile disaster: 2013 Typhoon Haiyan. The same information for the 2010 Haiti earthquake is available in [22] but was omitted here for brevity. From the description and a number of policy documents and guidelines provided by DROs, we extract structured elements such as the different actors and their activities which we finally pour into a novel generic mobility model.

\subsection{Scenario Selection Criteria}

Table 2 lists recent natural disasters and their scale. We found that the following six factors are key for the creation of scenarios and models for DTN research in the area of natural disasters: (1) the number of affected people and (2) the size of the affected area both define the scope of the disaster; (3) geographic conditions are important as impassable natural obstacles such as rivers or lakes would partition the network and render a DTN deployment unusable; (4) the availability of infrastructure (bridges, airports, power plants, cellular network, etc.) after the disaster; and (5) strong international response by DROs as they are the sources for (6) sufficient, finegrained information on the event. The latter is the most important factor for us as we base our model and scenario design solely on written and oral reports. Note that we did not include all presented factors in Table 2 due to space reasons, but provide an extended version in [22].

\subsection{Typhoon Haiyan}

We chose to re-create human mobility of the 2013 Typhoon Haiyan as media coverage and response was high, and communication infrastructure was dysfunctional during the first days. In this section, we will give an overview of the disaster and describe the situation in detail during the first week (days zero to six). The following 
Table 2: Large-scale natural disasters in the last ten years. Ratings range from low $(*)$ to high (***). Our scenarios are printed in bold. We selected Typhoon Haiyan as the running example for this paper.

\begin{tabular}{|c|c|c|c|c|c|c|c|c|}
\hline Disaster & YEAR & Country & Killed & INJURED & DisPlaced & $\operatorname{ArEA}\left(\mathrm{km}^{2}\right)$ & REsponse & INFORMATION \\
\hline Nepal earthquake & 2015 & Nepal & 9000 & 22000 & 2600000 & 3610 & $* * *$ & $* * *$ \\
\hline Cyclone Pam & 2015 & Vanuatu & 24 & 30 & 3300 & 12190 & $* *$ & $* *$ \\
\hline Ludian earthquake & 2014 & China & 617 & 2400 & 229700 & 1487 & $*$ & $*$ \\
\hline Typhoon Haiyan & 2013 & Philippines & 6300 & 28689 & 6000000 & 71503 & $* * *$ & $* * *$ \\
\hline Christchurch earthquake & 2011 & New Zealand & 185 & 2000 & - & 1426 & $* *$ & $* * *$ \\
\hline East Africa drought & 2011 & East Africa & 260000 & - & 5720000 & 2346466 & $* * *$ & $* * *$ \\
\hline Tropical storm Washi & 2011 & Philippines & 1292 & 2002 & 430900 & 104530 & $* *$ & $*$ \\
\hline Tohoku earthquake & 2011 & Japan & 15894 & 6152 & 340000 & 83955 & $* *$ & $* * *$ \\
\hline Haiti earthquake & 2010 & Haiti & 316000 & 300000 & 895000 & 27750 & $* * *$ & $* * *$ \\
\hline Afghanistan blizzard & 2008 & Afghanistan & 926 & 100 & - & 652864 & $*$ & $*$ \\
\hline Sichuan earthquake & 2008 & China & 69195 & 374643 & 5000000 & 485000 & $* *$ & $* * *$ \\
\hline Cyclone Nargis & 2008 & Myanmar & 138000 & - & 800000 & 676578 & $* *$ & $* *$ \\
\hline
\end{tabular}

information was gathered through interviews ${ }^{1}$ with multiple first responder eyewitnesses from different relief organizations, as well as press articles about the relief efforts.

Summary. Typhoon Haiyan lasted from November 3rd to 11th, 2013 and was one of the strongest tropical cyclones ever recorded [18]. Even though typhoon Haiyan had devastating effects on large portions of Southeast Asia, for the purpose of this work we will focus on the aftermath of November 8th when Typhoon Haiyan hit the Philippines at 04:40 local time. Haiyan was the deadliest and most damaging Philippine typhoon on record and left more than one million houses partially or totally damaged, killing at least 6300 people and leaving numerous injured and homeless [15]. Typhoon Haiyan was ranked as a category 5 typhoon, the highest category by the definition of the Saffir-Simpson Hurricane Wind Scale (SSHWS), implying that "catastrophic damage will occur" and "most of the area will be uninhabitable for weeks or months." After the storm had passed, widespread damage became visible with power lines cut off, roads blocked by fallen debris, and trees and buildings collapsed under the strong winds [19]. A 2013 preliminary estimate [14] calculated the total damage related to typhoon Haiyan to be around 2.86 billion US\$. Figure 2 depicts the city of Tacloban and its surroundings, which were severely hit by Typhoon Haiyan, and is intended to provide visual guidance to the reader for a better understanding of the upcoming sections.

Right after the disaster had hit the Philippines, many officials concluded that even though early warnings had been issued to the population of potentially affected areas, only few people actually evacuated. This was likely related to the high number of smaller typhoons the Philippines experience every year, which led to the population underestimating the severity of the coming typhoon. Warnings were broadcast on TV and radio, but went largely unheeded. The typhoon was accompanied by the biggest storm surge ever experienced within the Philippines, reportedly reaching between four to six meters in height [24]. This resulted in fast-rising tides and surge water brought by the typhoon, which led to many additional fatalities [4].

\footnotetext{
${ }^{1}$ Interview notes are available upon request.
}

Day 0. In the aftermath of the typhoon, the immediate arrival of DRTs was hindered by the severe damage the airport had sustained. Therefore, many DRTs were rerouted to the airport of the nearby island of Cebu, which was still operational. From there, they had to travel to Tacloban using other means, which took around 8 hours. This slowed down the initial arrival of DRTs considerably. Despite the damage, a number of airplanes and helicopters managed to land in Tacloban, delivering aid and personnel. The newly arrived DRTs registered at the Reception/Departure Centre (RDC) before proceeding into the city to reach the On-Site Operations Coordination Centre (OSOCC). There were also a number of storm chasers and typhoon experts already on the ground when Haiyan made landfall. In search of scientific evidence, they moved through the city before, during and after the typhoon. (For our mobility model, we will assume that they are distributed randomly throughout the city.) The local population spent the storm in their homes or shelters. After the storm had passed, eyewitnesses report that most of the population seemed to wander around the perimeter of their home or shelter area, overwhelmed by the destruction caused by the storm, or trying to help their neighbors. (For our mobility model, we will assume their locations to be randomly spread throughout the city.) At the end of the day, DRTs and experts alike returned to their respective sleeping spots.

Day 1 to 3. On the first day after the disaster, the US Navy deployed radar equipment to the Tacloban airport, allowing it to be re-opened in short order. This allowed a greater number of DRTs and relief goods to arrive close to the city, leading to more travel between the airport and the city. Arriving DRTs reported at the RDC and then proceeded to the OSOCC, which was located in the city center. Afterwards, they travelled to the base camp of their organization and began their relief efforts. The initial focus of most DRTs lies with Search and Rescue Operations (SROs), as the likelyhood of finding survivors drops with every hour. Urban Search and Rescue Teams (USRTs) have a large number of different search strategies. (For the purpose of the movement model, we implement a basic strategy: a start area is chosen, and the team searches every house along that street before spreading out to the next street in 


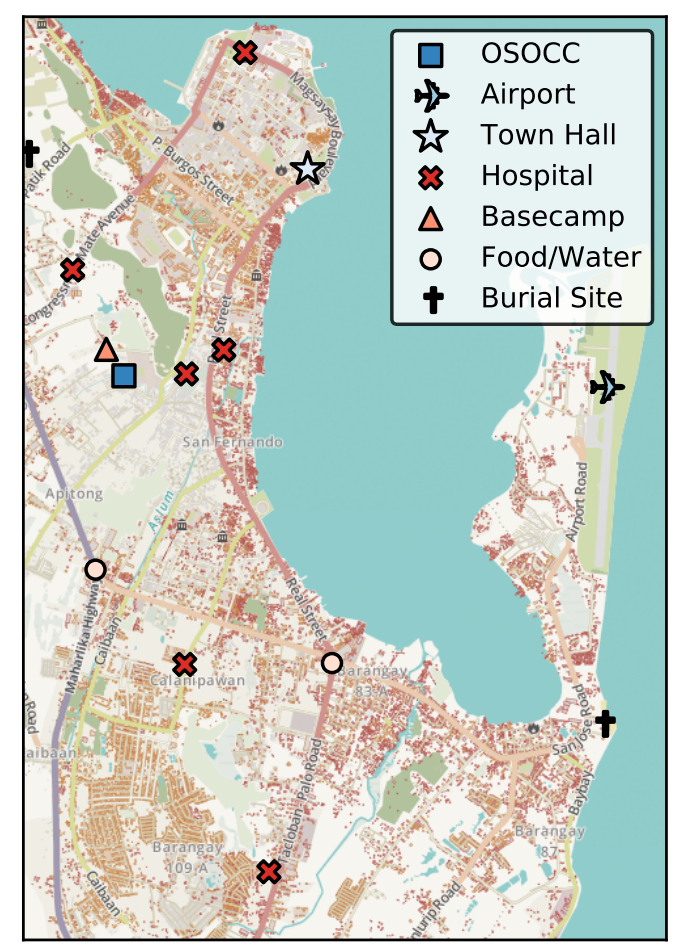

Figure 2: City of Tacloban with locations of IDR-relevant sites in the early aftermath of 2013 Typhoon Haiyan.

the direct neighborhood.) Eyewitnesses indicate that during the first three days, the civilian population was mostly concerned with search for food and the rebuilding of their homes or temporary shelters. Healthy survivors assisted with local search and rescue efforts and cleared the street from debris, staying in the general area of their homes or shelters. Injured survivors were transported to one of the partially operational city hospitals, which were quickly filled to capacity. As the government still remained in office, the relief organizations had to coordinate with country officials. This led to some traffic between the city hall and OSOCC. The United Nations (UN) rented a hotel as a temporary office location for the duration of the rebuilding efforts. ${ }^{2}$ All DRTs continued to return to their respective basecamps for the night.

Day 4 to 6. SROs were reduced and came to a stop at the end of the first week, as the chance of finding survivors drops significantly after several days. After about one week, most of the USRTs are replaced by teams specialized in other forms of disaster relief. The distribution of food and clean water remained a challenge, as the infrastructure was severely damaged by the typhoon. Much of the local population had to collect food and water from distribution points around the city on a daily basis. The local population assisted DRTs in clearing the roads of debris to allow trucks to pass through them. The government also ordered the removal of dead bodies to prevent the spread of diseases. The US Marines started flying out injured locals and exhausted relief workers alike. At the same time,

${ }^{2}$ We were asked not to publish the location of the hotel, so we randomly chose a hotel in the city for the purposes of the simulation. new DRTs were still arriving via the airport, following the same procedure as previously described.

After Week 1. We stop our description of the events after the first week. At this time, locals had slowly begun to resume a semblance of their normal lives, and the scenario gradually transforms into a scenario of daily routine, where a dedicated mobility model no longer applies. For the same reason, we did not consider that most DROs have a rotation mechanism for their employees, which prevents them from becoming mentally and physically exhausted from the demanding work in disaster relief.

\subsection{Natural Disaster Mobility Model}

Based on the information gathered for specific disasters, we extract recurring behavioral patterns of the various entities involved in disaster relief work. To this end, we define roles and role-specific activities, and also consider movement speed.

Roles. We identified the main stakeholders in natural disasterstruck areas (scenario-independent) and defined the following seven roles with distinct behavioral patterns: (1) healthy local population, (2) injured local population, (3) Disaster Response Teams (DRTs) from DROs, (4) dedicated Urban Search and Rescue Teams (USRTs), (5) scientists (storm chasers, typhoon experts, etc.), (6) UN officials, and (7) government officials.

Activities. To create a model, we further need to define activities that regularly occur in disaster areas that can be attributed to specific roles. Figure 3 summarizes the various activities by showing the coarse locations of each role during the course of the first week. What follows is a detailed but non-exhaustive description of important activities that have been identified during the interviews with IDR experts.

Activities applying to everyone. In the evening, everyone goes to their respective base camp, home, or shelter to sleep. Those arriving via the airport (e. g., DROs), at the day of arrival, first go to the RDC for registration, then visit the OSOCC, and finally set up the base camp or sleeping place.

Activities applying to DROs and DRTs. After arriving and registering at the airport, they go to the OSOCC or the town hall for a situation briefing and then start to help the affected population with one of the following activities: $(i)$ collect dead bodies and organize burials; (ii) walk the main streets of the city and clean streets from debris, such that supplies can be delivered; or (iii) go to food and water distribution centers to serve the locals until the end of the day. Besides that, they regularly visit the UN hotel, the OSOCC site, the base camp, or town hall to meet with officials and other DROs.

Activities applying to UN and government officials. Officials regularly (at least daily) visit the OSOCC, the town hall or base camp, for a situation briefing and meet other officials and DROs. During the day, they perform reconnaissance missions to get an situation overview such as infrastructural damage. This information is used to provision help. Also, they organize the disaster relief efforts with other officials and DROs such as setting up food and water distribution spots, organizing burials, etc.

Activities applying to scientists. Within the first two to three days, they collect scientific evidence from the disaster site before the cleaning of the rubble and debris starts. When their job is done, 


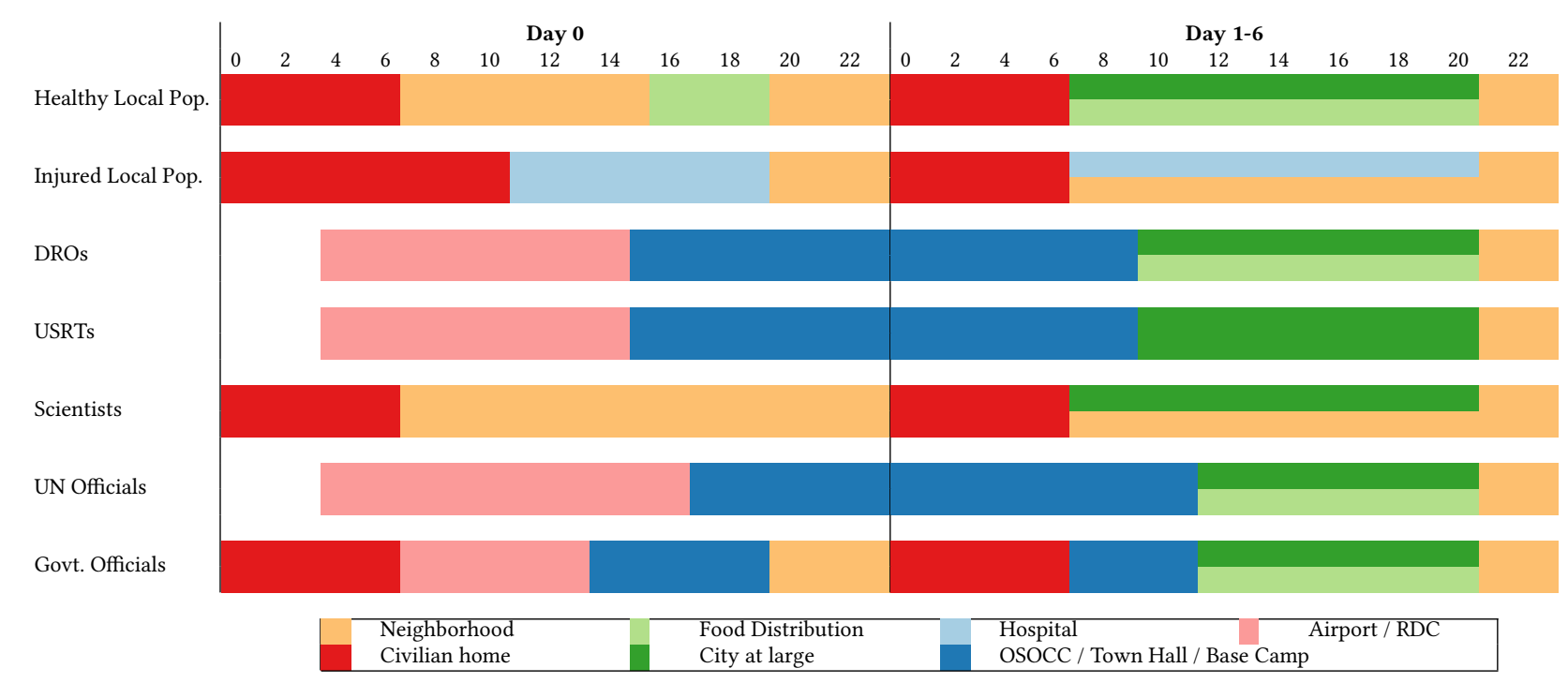

Figure 3: Activity-based location of different groups over time. Time values are indicative, i. e., activities do not start exactly at the same time but with a random jitter of one or two hours.

they either leave the area via the airport, or decide to volunteer and help the DROs.

Activities applying to USRT. After arriving and registering at the airport, they go to the OSOCC for a situation briefing and SRO planning. When starting an SRO, USRTs go to the chosen location in the morning and then search every house in that street. When done, they repeat with next street in the direct neighborhood. Usually, SROs are stopped after one week as the chances to find survivors diminish, and USRTs fly back home.

Activities applying to healthy local population. According to eye witnesses, most locals stay at home or try to find friends and family members within the immediate surroundings after the disaster has struck. Later on the first day, they stay in the proximity of their homes to assess the damage and to help their neighbors. Then, they start to look for food and water, for example, at distribution centers where they will return on a daily basis. The rest of the day, they either volunteer for cleaning operations (we model this by slowly roaming around the city), or as replacement of security personnel to patrol the area.

Activities applying to injured local population. Depending on the severeness, the injured stay at home if they are unable to move, or try to go to the closest hospital as soon as possible. Upon arrival, they stay at the hospital if its capacity is not exhausted or leave to find another one otherwise.

Typhoon Haiyan Arrival Times. Most scientists, particularly storm chasers and typhoon experts were already present prior to the occurrence of the disaster. Furthermore, government officials were already present prior to the disaster, while UN officials arrived after the disaster had struck. DROs and USRTs mainly arrived via the airport during the first days after the disaster as they were delayed due to the damaged airport. However, some had been prepositioned to locations close to the affected area and arrived on day zero.

Movement Speed. For the purpose of this work, we consider walking the only viable form of movement. This is due to the fact

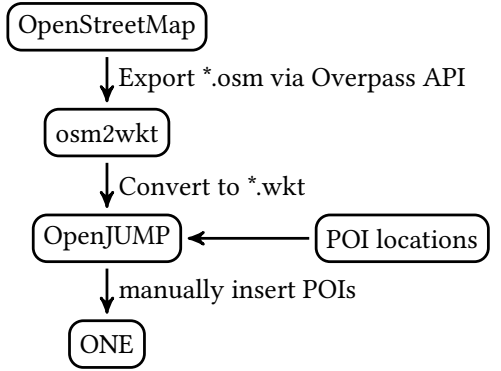

Figure 4: Technical workflow for generating a scenario's map data for our Natural Disaster mobility model.

that within disaster-struck areas, debris, flood/surge water, or earthquakes often render streets completely unusable for cars and trucks until they can be repaired. This means that all entities move approximately at walking speed. Nevertheless, there are slight differences depending on the entity's role. For example, injured individuals and heavily equipped USRTs will be slower than normal. Note that our model could be extended to support faster-traveling vehicles, but would require additional scenario-specific information currently unavailable to us, such as who has access to vehicles and which streets are passable.

\section{IMPLEMENTATION}

In this section, we briefly describe the implementation of our generic Natural Disaster mobility model and the workflow for generating specific scenarios. This is a trade-off between code re-usability (reoccurring behavioral patterns of certain groups such as DROs and the local population) and acknowledging the uniqueness of every disaster (terrain, streets, point of interests (POIs), arrival times, population density, etc.) 
Mobility Model. We implement our mobility model directly as a module for the Opportunistic Network Simulator (ONE) [8] as we are concerned with DTN simulation. Our model implements the different roles and activities as defined in Section 4 . These roles and activities have been observed in different disasters (Table 2) and as such are generally independent of the scenario instance. Nevertheless, our model can only operate on a specific scenario.

Scenario. A scenario describes the prevailing conditions of a specific post-disaster situation. In particular, the scenario comprises the number of actors as well as a street map including different POIs of the affected area. We generate the map data using a number of tools shown in Fig. 4: we start with exporting OpenStreetMap data and converting it to the Well-Known Text (.wkt) file format using osm2wkt [13]. Finally, we use OpenfUMP to add POI locations, such as the OSOCC, airports, and hospitals as shown in Fig. 2. The .wkt files can then be processed by our mobility model implemented in the ONE. In this paper, we only focus on the area around the city of Tacloban which was severely hit by Typhoon Haiyan. However, we have also implemented a scenario for the 2010 Haiti earthquake (Port-au-Prince and surroundings) which is discussed in [22] and included in our source code [23]. Using our model, workflow, and explanations in [22], third parties are able to create additional scenarios. In addition, our implementation is open to extensions such as more detailed activities, vehicle support, etc.

\section{EVALUATION}

In this section, we want to $(i)$ visually validate our mobility model $(N D)$, (ii) identify qualitative differences between $N D$ and other contemporary models, (iii) assess the impact that different mobility models have DTN performance, and finally (iv) give actionable advice to DTN protocol designers. To this end, we compare $N D$ with two other widely used models: the Random Waypoint Mobility model (RWP) and a map-based RWP model (Map) where waypoint selection is still random, but node movement is confined to a street grid. We selected the epidemic DTN routing protocol for the network performance analysis as its simple design facilitates reasoning about the results. For all experiments, we rely on the ONE simulator v1.6.0 [8]. Each experiment is averaged over 10 independently seeded runs. We summarize the most important simulation settings in Table 3 and refer to our source code [23] for all details. For reproducibility, we also publish our experiment data set [21].

We note the limitation of our simulation setup with only 500 nodes. Even though our mobility model supports an arbitrary number of nodes, current network simulators (ONE, ns-2/3, etc.) do not scale to a large number of nodes, e. g., the population size in an urban area (order of $10^{6}$ ). However, as we will show, we can demonstrate qualitative differences between the different mobility models already with a rather low number of nodes. We also note that in our traffic model, sources and destinations as well as messages sizes are arbitrarily chosen. A realistic communication model would be incredibly useful but is orthogonal to our work.

\subsection{Characterizing Mobility}

We are interested in the spatial node distribution and encounters that occur during a disaster since they both affect the applicability of a DTN: node hot spots can function as communication hubs
Table 3: The ONE Simulation Settings

\begin{tabular}{rll}
\hline \multirow{2}{*}{ Scenario } & Dimensions & $5000 \times 7000 \mathrm{~m}^{2}$ (Tacloban) \\
& Simulation time & 7 days \\
& Nodes & 500 \\
\hline \multirow{2}{*}{ Mobility } & Model & RWP, Map, ND \\
& Speed $(\mathrm{m} / \mathrm{s})$ & $0.5-1.5 \approx$ walking speed \\
\hline \multirow{2}{*}{ Routing } & Algorithm & Epidemic \\
& Buffer size & $20 \mathrm{MB}$ \\
\hline \multirow{2}{*}{ Taffic } & Msg. interval & $8-12 \mathrm{~s}$ \\
& Msg. TTL & $6 \mathrm{~h}$ \\
& Msg. size & $50-100 \mathrm{~KB}$ \\
\hline \multirow{2}{*}{ Comm. } & PHY rate & $2 \mathrm{Mbit} / \mathrm{s} \approx$ Bluetooth \\
& Radio range & $10 \mathrm{~m}$ \\
\hline
\end{tabular}

where messages are quickly exchanged, while nodes that have a lot of encounters can act as "data mules" and transport messages over larger distances.

Spatial Node Distribution. We visualize the spatial node distribution of the three mobility models using a scatter-plot heatmap in Fig. 5. In Fig. 5a, we identify the typical non-uniform centerweighted distribution [3] of the RWP model. From a practical perspective, this means that nodes are moving across inaccessible areas, for example, a bay. In contrast, Map and $N D$ (Figs. 5b and 5c) essentially "redraw" the underlying street grid. Here, the nodes' movements are confined to streets and paths and are thus no longer moving across water. However, node distribution in Map across streets appears generally uniform. There are only minor hot spots at street intersections which are expected since movement trajectories cross there. In general, nodes are located with similar probabilities at any point in the map. Figure $5 \mathrm{c}$ shows that $N D$ exhibits characteristic hot spots that can be mapped to certain POIs in the street map (Fig. 5d) where many nodes stay for a longer period of time. Most prevalent are the locations of the OSOCC and the base camp as they are frequented by DRTs and officials. In addition, we can also make out other hot spots at the city hall and the food and water distribution points. In the simulation, the airport is the location for inactive nodes, that is, those nodes that have not yet arrived and those that have already left. The hot spots around the airport can therefore be considered an artifact as the simulator does not support removing nodes from a running simulation.

Encounters. An encounter is a transmission opportunity which occurs if two nodes move in each other's transmission range. DTNs performance highly depends on the number of encounters a node makes while moving around. For example: if a node encounters the destination of any currently carried message, it can directly deliver it. The advantage of direct delivery is that it prevents the replication overhead to intermediate nodes in form of radio transmissions and storage consumption. So, in a scenario where communicating parties are generally physically close to one another, or at least meet regularly, a DTN deployment could exclusively rely on direct deliveries. Therefore, assessing the encounter characteristics of the underlying mobility model in a scenario is of utmost importance to understand which protocols are suitable for a natural disaster scenario. In Fig. 6, we observe that in $N D$, the local population groups (healthy and injured) make significantly fewer contacts 


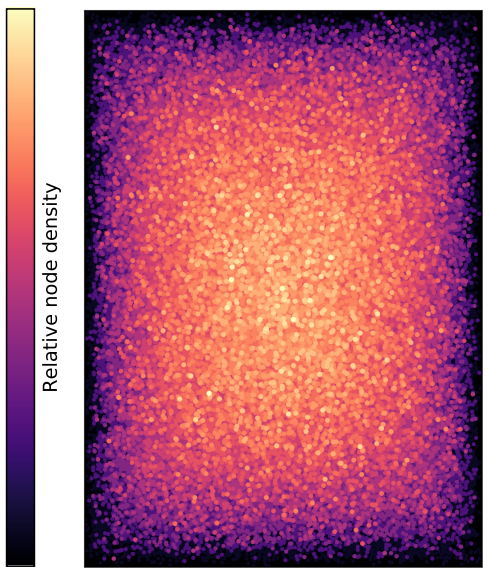

(a) RWP

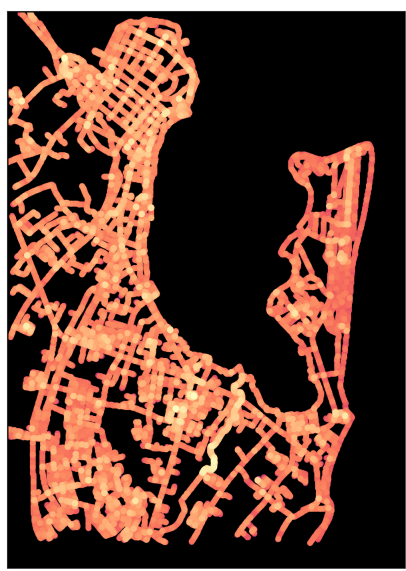

(b) Map-based RWP

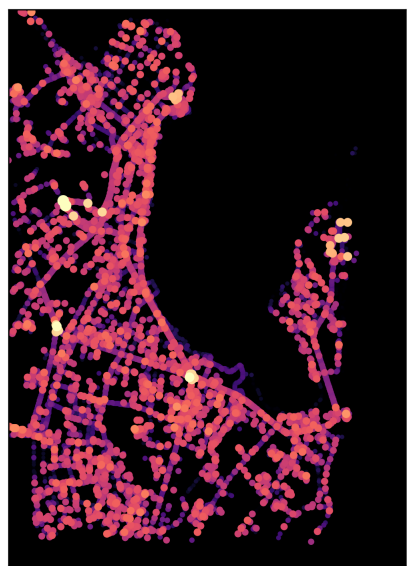

(c) Natural Disaster

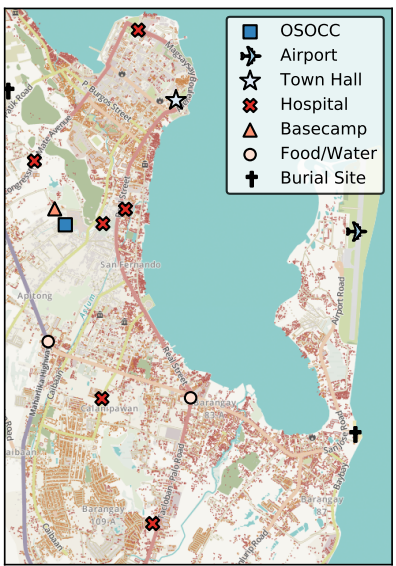

(d) Reference map (same as Fig. 2)

Figure 5: Spatial node distribution in different mobility models averaged over 7 days. Node counts are sampled from a grid of $10 \times 10 \mathrm{~m}$ squares. Circle sizes and colors scale logarithmically with node count to highlight hot spots.

than the other groups. Especially the injured encounter very few other nodes. On the other hand, DRO teams as well as government and UN officials make significantly more contacts due to regular meetings at the OSOCC, in the town hall, and in the base camps. In the RWP and Map models, the number of encounters solely depends on the average velocity of the user role. For example, injured as well as heavily equipped USRTs move slower than the other groups. The generally low number of encounters of $R W P$ can be explained by the low node density in combination with the low transmission range: as the nodes freely move around the large area, nodes only infrequently move into each others transmission range.

\subsection{Characterizing Network Performance}

We evaluate the impact of our mobility model on network performance. For this, we measure the delivery rate and delay, the buffer occupancy, as well as the delivery rate for the different roles. In Fig. 7, we observe that the delivery delay short in $N D$, meaning that about $80 \%$ of all delivered messages are delivered within three hours, which appears to be due to the regular meetings at certain POIs. However, we can see in Fig. 9 that delivery success is highly heterogeneous with respect to the user role in $N D$. We observe that the most successful communication partners are the

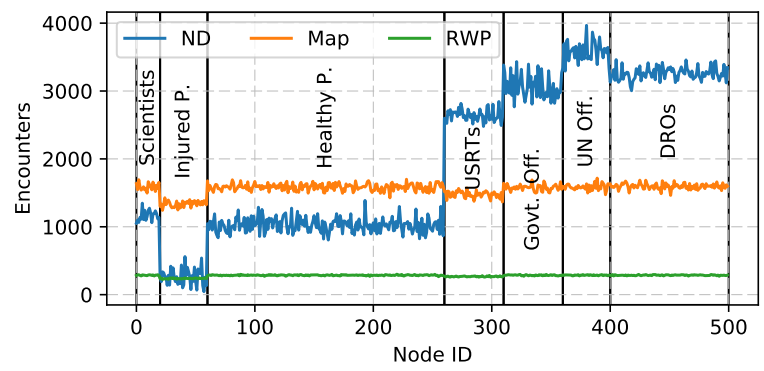

Figure 6: Number of encounters per node (1 week). ones that make the most encounters, in particular, DROs, USRTs, and government/UN officials. These are the groups that can rely on message deliveries the most and are, thus, the main beneficiaries of the communication network. On the other hand, less mobile nodes, especially the injured population, are poorly connected to the rest. Without special treatment (e. g., via prioritization), these users will be insufficiently served by the network. In RWP and Map, performance is almost uniform across the different group (not shown here). The overall delivery success of $N D$ is lower than for Map which is likely due to the fact that at night, when mobility is low, only few messages are delivered. This is supported by Fig. 8 which shows that buffer occupancy is depending on the time of day. During the night, undelivered messages expire and nodes remove them from their buffers. Starting around noon, buffers refill with new messages as nodes travel across the map and collect messages from others, leading to more message deliveries during the day. Note that the delivery success over time is not shown here for space reasons, but follows the same cyclic behavior as the buffer occupancy.

\section{CONCLUSION}

DTNs could greatly facilitate disaster response communications as they allow message-based communication even in the absence of supporting infrastructure. However, so far, the communication community has yet to prove the practicality of DTNs for such scenarios. In this paper, we have shown the feasibility of reverse engineering human mobility solely by written or oral reports and have extract general as well as scenario-specific features and patterns. Based on two exemplary scenario, we were able to assess the performance of DTNs in large-scale natural disasters as a backup for infrastructure-based communication, and develop a tool for future works in this area. In particular, we observed that a DTN deployment could be most useful for disaster relief workers due to regular meetings, while the population benefits to a lesser degree. Based on these observations, we give advice to DTN designers such as preferring frequently traveling nodes as relays and exploiting hot spots 


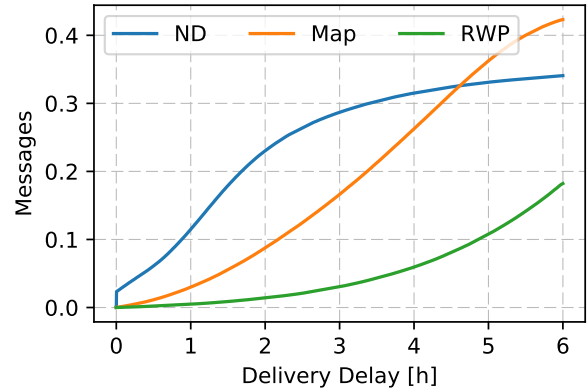

Figure 7: Message delivery success and delay as a cumulative distribution function.

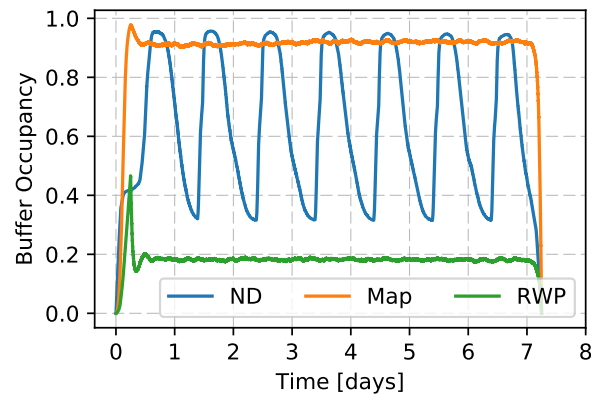

Figure 8: Buffer occupancy over time.

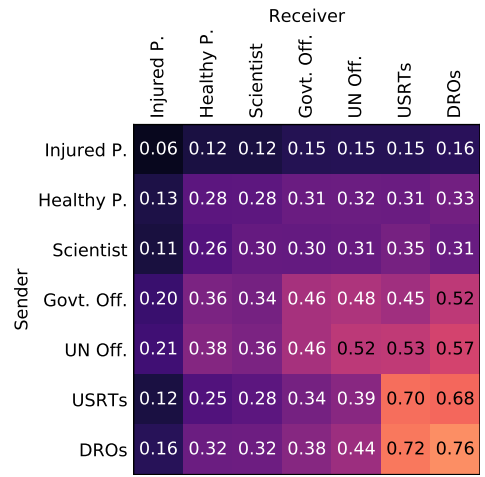

Figure 9: Delivery rates between roles $(N D)$. by deploying fixed DTN nodes to serve as information exchange hubs. In the future, we could increase the level of detail in our mobility model, for example, by including vehicles. Our network performance evaluation could be extended, which would require to increase the scalability of current simulators to support simulations with significantly more nodes. Finally, a realistic communication model of all users in a disaster scenario would complement our mobility model since communication patterns and user mobility are the two main factors influencing DTN performance.

\section{ACKNOWLEDGMENTS}

The authors would like to express their sincere gratitude towards all cooperating individuals from the following national and international organizations: Caritas (AT), Berufsfeuerwehr Bochum (DE), Johanniter (DE), Red Cross (DE), Technisches Hilfswerk (DE), emergency.lu (LU), Humanitarian Intervention Team (LU), Ministry of Foreign Affairs (LU), Concern Worldwide, Plan International, ZOA International, Emergency Telecommunication Cluster (ETC), Global Disaster Alert and Coordination System (GDACS), Office for the Coordination of Humanitarian Affairs (OCHA), United Nations Organizations (UNO), United Nations Office for Disaster Risk Reduction (UNISDR), World Food Program (WFP), and many others who wished to remain anonymous.

This work has been co-funded by the LOEWE initiative within the NICER project, and by the DFG as part of project C.1 within the RTG 2050 "Privacy and Trust for Mobile Users."

\section{REFERENCES}

[1] N. Aschenbruck, M. Frank, P. Martini, and J. Tolle. 2004. Human Mobility in MANET Disaster Area Simulation - A Realistic Approach. In IEEE LCN.

[2] N. Aschenbruck, E. Gerhards-Padilla, and P. Martini. 2009. Modeling mobility in disaster area scenarios. Elsevier Performance Evaluation 66, 12 (2009).

[3] C. Bettstetter, G. Resta, and P. Santi. 2003. The node distribution of the random waypoint mobility model for wireless ad hoc networks. IEEE Transactions on Mobile Computing 2, 3 (July 2003), 257-269.

[4] Dailymotion. 2013. Megastorm: Worlds Biggest Typhoon. (2013). http: //www.dailymotion.com/video/x186qfd_megastorm-worlds-biggest-typhoon_ shortfilms

[5] H. Goldstein. 2010. Engineers Race to Restore Communications after Haiti Quake. IEEE Spectrum (19 Jan. 2010).

[6] ICRC, The Engien Room, and Block Party. 2017. Humanitarian Futures for Messaging Apps. Technical Report.

[7] IRIN News. 2013. Life-saving radio begins broadcasting in typhoonhit Tacloban. (2013). http://www.irinnews.org/report/99132/ life-saving-radio-begins-broadcasting-typhoon-hit-tacloban

[8] A. Keränen, J. Ott, and T. Kärkkäinen. 2009. The ONE simulator for DTN protocol evaluation. In ICST Conference on Simulation Tools and Techniques (SIMUTools'09).

[9] S. Krug, S. Schellenberg, and J. Seitz. 2015. Impact of Traffic and Mobility Patterns on Network Performance in Disaster Scenarios. In ACM CHANTS.

[10] S. Krug, M. F. Siracusa, S. Schellenberg, P. Begerow, J. Seitz, T. Finke, and J. Schroeder. 2014. Movement patterns for mobile networks in disaster scenarios. In IEEE WoWMoM.

[11] J. Leaning. 2013. Natural Disasters, Armed Conflict, and Public Health. The New England Journal of Medicine (2013)

[12] A. Martín-Campillo, J. Crowcroft, E. Yoneki, and R. Martí. 2013. Evaluating opportunistic networks in disaster scenarios. Fournal of Network and Computer Applications 36, 2 (March 2013).

[13] C. P. Mayer. 2010. osm2wkt - OpenStreetMap to WKT Conversion. http://www.chrismc.de/osm2wkt. (2010).

[14] NDRRMC. 2013. Final Report - Effects on Typhoon "Yolanda" (Haiyan). (Nov. 2013). http://www.ndrrmc.gov.ph/attachments/article/1329/FINAL_REPORT_re Effects_of_Typhoon_YOLANDA_(HAIYAN)_06-09NOV2013.pdf

[15] NDRRMC. 2013. Situation Update - Effects of Yolanda. (2013). https://web.archive. org/web/20161208214400/http://www.ndrrmc.gov.ph/attachments/article/1177/ Update/\%20Effects/\%20TY/\%20YOLANDA/\%2017/\%20April/\%202014.pdf

[16] S. C. Nelson, A. F. Harris III, and R. Kravets. 2007. Event-driven, role-based mobility in disaster recovery networks. In ACM CHANTS.

[17] H. Nishiyama, N. Ito, and N. Kato. 2014. Relay-by-smartphone: realizing multihop device-to-device communications. IEEE Communications Magazine 52, 4 (2014).

[18] NPR. 2013. Why Typhoon Haiyan Caused So Much Damage. (2013). http://www. npr.org/2013/11/11/244572227/why-typhoon-haiyan-caused-so-much-damage

[19] International Charter on Space and Major Disasters. 2013. Activation - Typhoon Haiyan in the Philippines. (2013). https://www.disasterscharter.org/web/guest/-/ typhoon-haiyan-in-the-philippin- 5

[20] D. G. Reina. 2012. Modeling and assessing ad hoc networks in disaster scenarios. fournal of Ambient Intelligence and Humanized Computing (2012).

[21] M. Schmittner. 2017. Experimental Data for Natural Disaster Mobility Model and Typhoon Haiyan Scenario. (2017). https://doi.org/10.5281/zenodo.836815

[22] T. Schons. 2017. Design, Implementation and Evaluation of Realistic Scenarios and Movement Models for Natural Disasters Using Simulation for Delay Tolerant Networks. (Jan. 2017).

[23] T. Schons. 2017. Natural Disaster Mobility Model and Scenarios Source Code. (2017). https://github.com/seemoo-lab/natural-disaster-mobility

[24] The Telegraph. 2013. Super Typhoon Haiyan smashes into Philippines. (2013). http://www.telegraph.co.uk/news/worldnews/asia/philippines/10434846/ Super-Typhoon-Haiyan-smashes-into-Philippines.html

[25] M. Y. S. Uddin, D. M. Nicol, T. F. Abdelzaher, and R. H. Kravets. 2009. A postdisaster mobility model for Delay Tolerant Networking. In Winter Simulation Conference (WSC). 